\title{
Relación entre práctica y asistencia a eventos deportivos: un estudio sobre el caso del judo en España
}

\author{
Pau MATEU ${ }^{1} \&$ Eduard INGLÉS \\ Institut Nacional d'Educació Física de Catalunya (INEFC) - Barcelona (España) \\ Grup d'Investigació Social i Educativa de l'Activitat Física i l'Esport (GISEAFE) (España)
}

Recepción: 04/10/2016; Aceptación: 03/04/2017; Publicación: 04/04/2017.

\section{Resumen}

La presente investigación pretende mostrar el perfil de los asistentes a competiciones de judo en España, así como la relación entre práctica deportiva y asistencia a eventos de judo. La muestra se compuso por 270 asistentes a tres eventos de judo absoluto y sénior, de diferentes niveles competitivos. La recogida de datos se llevó a cabo mediante cuestionarios administrados durante la celebración de las competiciones estudiadas. Se realizaron análisis de frecuencias y porcentajes, comparaciones de medias entre sexos y procedimientos de contraste. La información obtenida expone los perfiles mayoritarios de las personas que asisten a competiciones de judo en España. Asimismo, establece un vínculo positivo entre práctica deportiva y asistencia a eventos de judo, del mismo modo que muestra una alta proporción de practicantes y ex-practicantes de judo en eventos de esta disciplina. Este estudio supone un primer paso en el ámbito de la asistencia a eventos de artes marciales y deportes de contacto en España, y se abre a la posibilidad de seguir estudiando sobre el tema, tanto en el caso del judo como en el de otras disciplinas.
\end{abstract}

ORIGINAL PAPER

Palabras clave: artes marciales; deportes de combate; eventos deportivos; asistentes; práctica deportiva; judo.

\section{Relationship between sport participation and attendance at sporting events. A case study on the judo in Spain}

\begin{abstract}
This research aims to show the profile of attendees to judo competitions in Spain, as well as the relationship between sports participation and attendance to judo events. The sample consisted of 270 people who attended three absolute and senior judo events of different competitive levels. Data collection was carried out through questionnaires which were administered during the celebration of the studied events. Frequency and percentage analysis, means comparisons between sexes and contrast procedures were performed. The information obtained shows the majority profiles of people attending judo competitions in Spain. It also establishes a positive link between sport participation and judo events attendance, just as it shows a high proportion of judo players and former judo players at events in this sporting discipline. This study is a step forward in the field of attending martial arts events and contact sports in Spain, and opens the possibility to continue studying on the subject, both in the case of judo so as in other disciplines.
\end{abstract}

Keywords: martial arts; combat sports; sport events; attendees; sport participation; judo.

\section{Relação entre a prática e a assistência em eventos desportivos. 0 estudo de caso do judo, em Espanha}

\section{Resumo}

A presente investigação pretende mostrar o perfil dos assistentes nas competições de judo, em Espanha, assim como a relação entre a prática desportiva e a assistência nos eventos desta disciplina. A amostra foi de 270 assistentes, em três eventos de judo absoluto e sénior, de diferentes níveis competitivos. Procedeu-se à recolha de dados através de questionários administrados durante a celebração das competições estudadas. Realizaram-se análises de frequências e de percentagens, comparações de médias entre sexos e procedimentos de contraste. A informação obtida expõe maioritariamente os perfis das pessoas que assistem a competições de judo, em Espanha. Deste modo, estabelece-se um vínculo positivo entre a prática desportiva e a assistência em eventos de judo, do mesmo modo que se revela uma alta proporção de praticantes e ex-praticantes de judo em eventos desta prática. 0 estudo supõe um primeiro passo no âmbito da investigação sobre a assistência/público de artes marciais e desportos de contacto, em Espanha, e abre a possibilidade de se continuar a estudar o tema, tanto no judo, como noutras disciplinas.

Palavras-chave: artes marciais; desportos de combate; eventos desportivos; assistentes; prática desportiva; judo.

\footnotetext{
1 E-mail: pmateu@gencat.cat. Address: Institut Nacional d'Educació Física de Catalunya (INEFC) - Barcelona. (Laboratorio de investigación social y educativa). Av. de l'Estadi 12-22. 08038 Barcelona (España).
} 


\section{Introducción}

En los últimos años, la importancia económica del deporte en España ha crecido de manera significativa (Benítez \& Lacomba, 2012) y está íntimamente relacionada con el crecimiento de la participación deportiva (Lera \& Suárez, 2012). En el caso de España, la participación deportiva ha evolucionado del 34\% en 1985 al 53,5\% en el 2015 (García \& Llopis, 2010; Ministerio de Educación, Cultura y Deporte, 2015). Del mismo modo, la asistencia a eventos deportivos también ha seguido una tendencia al alza (Lera \& Suárez, 2012), si bien existen grandes diferencias entre los deportes.

El judo es un deporte poco mediático, que se sitúa fuera de los diez deportes con más aficionados en España (Havas Sports \& Entertainment, 2013). Fuentes de la asamblea de la Federación Valenciana de Judo han confirmado que el nivel de aficionados que asisten a ver competiciones de judo es bajo, a pesar de que la asistencia a estos eventos es gratuita (JLC, comunicación personal, 9 de enero de 2015). Sin embargo, el judo es un deporte que cuenta con 104.871 participantes federados (Consejo Superior de Deportes, 2015a), y la Real Federación Española de Judo y Disciplinas Asociadas obtiene la mayor parte de sus ingresos (59,6\%) a través de fondos propios (Consejo Superior de Deportes, 2015b). Esto dejaría en entredicho la percepción habitual de las federaciones deportivas, que asocian la subsistencia económica de un deporte al nivel de audiencia que dicha modalidad consigue atraer (Serrano, 1999).

Smith (2008, p. 34) se refiere a las personas que asisten a eventos deportivos como consumidores de deporte, considerándolos como "un individuo o grupo que utiliza el deporte o productos/servicios asociados al deporte a cambio de un pago directo (dinero) o indirecto (selección de un canal de televisión)". De acuerdo con este autor, los consumidores deportivos se diferenciarían en cuatro categorías: 1) consumidores de bienes deportivos, que compran productos deportivos como, por ejemplo, ropa deportiva, libros, suplementos nutricionales o productos licenciados (camisetas de un determinado equipo, etc.); 2) consumidores de servicios deportivos, que utilizan un servicio o experiencia relacionada con el deporte, lo cual no implica su participación directa en el acto deportivo; 3) participantes y voluntarios del deporte, quienes se implican directamente en el deporte o participan sin ser retribuidos en labores de apoyo para una determinada organización; y 4) animadores, espectadores y aficionados, que asisten a eventos deportivos en vivo, o bien los siguen por televisión, internet, etc.

Otros autores (Mason, 1999) equiparan los términos de asistente a eventos deportivos con el de aficionado: personas que, de forma habitual, compran entradas y acuden a eventos deportivos $\mathrm{y}$, cuando no asisten, muestran su afinidad hacia un equipo o jugador viendo dichos eventos por televisión o bien comprando objetos y/o ropa vinculados a dicho equipo/deportista. Partiendo de las definiciones expuestas, en un estudio acerca de modelos de asistencia a eventos deportivos aplicado al fútbol, Quispe (2013) expone que la asistencia a un evento deportivo viene condicionada por cuatro grupos de variables: 1) económicas-demográficas: ligadas a la economía (precio de entradas, ingresos de las organizaciones...), información de la población de una determinada ciudad, salario promedio, etc.; 2) propias del juego: agrupan las condiciones generales del juego (temperatura, clima, distancia del asistente al lugar de celebración del evento deportivo, si el evento es televisado...), las características del equipo/deportistas (jugadores internacionales, rivales, historia del equipo, etc.) y los servicios brindados en el mismo evento o sus anexos (plazas de aparcamiento, conexiones de transporte, servicio de bar...); 3) propias del aficionado: incluyen conductas, actitudes, sensaciones e intenciones de los aficionados que acuden a eventos deportivos; y 4) propias del equipo: aquellas ligadas a los resultados previos del equipo, su nivel de juego, posición en un determinado campeonato, incertidumbre en el resultado, etc.

Por otra parte, en un estudio sobre 40 actividades recreacionales y deportivas, Lera y Rapún (2006) muestran cinco perspectivas que pueden afectar a la asistencia de eventos deportivos: 1) el atractivo del juego, que incluye habilidades individuales, presencia de jugadores famosos, posibilidad de batir récords o entretenimiento; 2) la conveniencia del horario, esto es, día y hora de celebración del evento, clima, etc.; 3) la economía y el marketing, lo que implica el precio de las entradas, promociones, disponibilidad de formas de entretenimiento sustitutivas, retransmisión televisiva del evento...; 4) la socio-demografía, abarcando la edad, género, ocupación o edad, de la población; y 5) los factores de motivación como la mejora de la autoestima, los lazos familiares o la necesidad de pertenencia a un grupo. 
Son varios los autores que hablan de la importancia de conocer los motivos de asistencia eventos deportivos. Smith (2008) señala que el consumidor de deporte asiste a partidos, sigue ciertos deportes y compra determinados productos deportivos debido a razones diversas, las cuales no siempre responden a comportamientos racionales y pueden apreciarse desde el punto de vista económico, psicológico, socio-cultural, y de auto-concepto. Por otro lado, Funk (2008) habla de la motivación como un proceso que va desde la detección de la necesidad hasta el comportamiento de logro/adquisición de aquello deseado. Como factores que influyen en este proceso se encuentran la socialización, el rendimiento, la excitación, la estima y la diversión.

La mayor parte de la literatura relativa al análisis de las características y los motivos de los asistentes para acudir a eventos deportivos se centra en grandes eventos, especialmente aquellos en los que la taquilla representa una importante fuente de ingresos para las organizaciones deportivas, como es el caso del fútbol, el baloncesto, el automovilismo, etc. (Hall, O'Mahony, \& Vieceli, 2010; Molina, 2002). Del mismo modo, pero en menor cantidad, se han realizado estudios acerca de asistentes a eventos de menor dimensión económica y estructural, tales como las carreras de caballos, el fútbol americano indoor o los dardos (Daniels \& Norman, 2005; Greenwood, Kanters, \& Casper, 2006; Wasserberg, 2009). Por su parte, Wann, Grieve, Zapalac y Pease (2008) hallaron diferencias en los motivos de los asistentes que acuden a ver deportes individuales y colectivos. Dichos autores muestran que en los deportes individuales prima la motivación alrededor de aspectos estéticos, mientras que en los deportes de equipo predominan motivos asociados al entretenimiento, la autoestima o la pertenencia a un grupo.

En el ámbito concreto de las artes marciales y deportes de contacto, destacan las aportaciones de Kim et al. $(2009,2008)$, con sus propuestas de análisis del perfil de asistentes a eventos de MMA - acrónimo del deporte de combate conocido como artes marciales mixtas. En su propuesta, los autores utilizan un instrumento de observación diseñado específicamente para asistentes a eventos de deportes de combate, adaptado a partir de cuestionarios de estudios genéricos anteriores (Fink, Trail, \& Anderson, 2002; Funk, Mahony, \& Ridinger, 2002; Trail \& James, 2001; Wann, 1995). Para analizar a los asistentes a eventos deportivos de un deporte individual de combate, proponen observar cinco elementos: motivos para asistir al evento, características demográficas, experiencias previas, preferencias y comportamiento con los medios de comunicación. No se tiene constancia de ningún estudio específico acerca del perfil de los asistentes que acuden a eventos de judo.

Un aspecto que se ha revelado de especial interés en el estudio sobre la asistencia a eventos deportivos es la posible relación entre dicha asistencia y la práctica deportiva. A este respecto, la literatura muestra opiniones contrapuestas. Así, una corriente de trabajos a nivel internacional ha mostrado que ambos factores no están relacionados (Stone, 1971; Shamir \& Ruskin, 1984; Burnett, Menon, \& Smart, 1993; Lines, 2007). Sin embargo, también han sido varios los autores y organismos que han encontrado una vinculación positiva en la relación entre asistencia a eventos deportivos y práctica deportiva (Sloan, 1989; Milne \& McDonald, 1999; Thrane, 2001; Dawson \& Downward, 2009; Weed, 2009; Perényi, 2010; UK Sport, 2011). En España, los estudios que relacionan la práctica de ejercicio físico y la asistencia a eventos deportivos no son numerosos, si bien podemos encontrar tanto trabajos que no establecen relación entre ambos elementos (Lera \& Rapún, 2006), como otros que sí determinan un vínculo positivo entre práctica deportiva y asistencia a eventos deportivos (Serrano, 1999; García \& Llopis, 2010; Lera \& Suárez, 2012).

En uno de los estudios sobre los hábitos deportivos de los españoles (García \& Llopis, 2010), se informa de que más de la mitad de las personas que hacen deporte en España acuden (con mayor o menor frecuencia) a presenciar eventos deportivos. Del mismo modo, destaca que hasta un $65 \%$ de las personas que afirman no realizar práctica deportiva nunca asisten a este tipo de espectáculos. En este estudio, del mismo modo que en todos los mencionados con anterioridad, no se tiene en cuenta la disciplina practicada ni a qué clase de eventos deportivos se refieren. No se ha encontrado referencia alguna en cuanto a la relación entre asistencia a un acontecimiento deportivo y la práctica de la disciplina concreta que se acude a ver.

A partir de estas consideraciones, y con ánimo de generar un conocimiento que contribuya al mejor entendimiento de cuestiones relacionadas con la gestión de las artes marciales (Vertonghen \& Dortants, 2013), el objetivo de esta investigación fue conocer el perfil general del 
asistente a eventos de judo de categoría absoluta, y conocer en qué medida la práctica deportiva guardaba relación con la asistencia a eventos de este deporte. Este objetivo se plasmó en tres preguntas de investigación relacionadas entre sí:

1. ¿Cuál es el perfil del asistente a competiciones de judo absoluto en España?

a. ¿Qué proporción de practicantes de judo hay entre el público asistente a competiciones de judo absoluto en España?

b. ¿Cuál es el contexto social de práctica de los practicantes de judo que hay entre el público asistente a competiciones de judo absoluto en España?

A través de la primera pregunta se propone analizar los rasgos que caracterizan a las personas que acuden a ver competiciones de judo absoluto en España. Por otro lado, al responder a la segunda cuestión, se propone conocer, concretamente, cuál es el porcentaje de asistentes a eventos de judo absoluto que, a su vez, practican este deporte. Mediante la tercera pregunta, señalamos que no solo buscamos conocer el porcentaje de practicantes de judo entre los asistentes a sus eventos, sino también conocer información desde una perspectiva sociológica más amplia; en nuestro caso, dentro de los elementos que García y Llopis (2010) entienden por contexto social de la práctica deportiva. En este sentido, hemos seleccionado únicamente aquellas características que son relevantes en relación al objetivo planteado: si practican o no deporte, si este deporte practicado es el judo, con qué frecuencia lo practican, a qué tipo de organización deportiva pertenecen, a qué nivel competitivo lo practican y/o si poseen licencia federativa.

Por otro lado, se ha planteado una hipótesis que señala que la proporción de practicantes de deporte entre los asistentes a eventos de judo en España es más elevada que la existente en la población general, mostrada en el estudio de García y Llopis (2010).

\section{Material y método}

La técnica de investigación seleccionada para la recogida de datos fue la encuesta por cuestionario. Se trata de un método cuantitativo, a través del cual se trataron de identificar los motivos de los espectadores para asistir a competiciones de judo, así como la relación que guardan con la práctica de actividad deportiva en general, y de judo en particular. Se consideró idóneo el uso de este método dado que, tal y como afirman Quivy y Campenhoudt (2005), se utiliza con el objetivo de analizar un fenómeno social delimitado a partir de la información que se refiere a los individuos de nuestra población de estudio, así como para interrogar a un gran número de personas. Debido a que involucra un gran número de personas interrogadas, las respuestas a la mayor parte de preguntas están pre-codificadas para que los encuestados elijan sus respuestas entre las opciones que se les proponen (Quivy \& Campenhoudt, 2005).

Por otra parte, cabe tener en consideración las limitaciones de este método: las respuestas acostumbran a ser superficiales, se consideran las personas como independientes de sus redes de relaciones sociales y, además, la fiabilidad es frágil, por lo que se deben elegir rigurosamente la muestra, las preguntas de investigación, la correspondencia entre el mundo de referencia de las preguntas y el de las personas encuestadas, la atmósfera de confianza en el momento de administrar el cuestionario y la profesionalidad de los encuestadores (Quivy \& Campenhoudt, 2005).

\subsection{Instrumento}

Se elaboró un cuestionario ad hoc para la presente investigación, compuesto por un total de 53 ítems. Este cuestionario combina las propuestas de García y Llopis (2010) y Kim et al. (2009, 2008), y está diseñado para recopilar información relativa a:

- Características sociodemográficas (6 ítems): preguntas cerradas y pre-codificadas acerca del sexo, edad, estado civil, nivel de estudios, situación laboral y la nacionalidad de los participantes (García \& Llopis, 2010; Kim et al., 2009). 
- Motivos (33 ítems): se adaptó la escala de Kim et al. (2009) para MMA. Los encuestados evaluaron tres afirmaciones para cada uno de los once motivos estudiados, mediante escalas de Likert de siete puntos, entendidos como (1) Muy en desacuerdo y (7) Muy de acuerdo. Los motivos analizados fueron: 1) drama, los asistentes se muestran excitados por un evento emocionante o por combates en los que no se puede predecir un resultado; 2) escape, los espectadores acuden al evento para olvidar temporalmente sus problemas, o para revitalizar su vida escapando de la rutina; 3) calidad estética, los asistentes conocen las técnicas de lucha y disfrutan de combates entre competidores; 4) logros vicarios, mirar los combates ofrece a los asistentes una sensación que no pueden lograr en su vida actual; 5) socialización, los eventos deportivos son utilizados para hacer amigos o pasar tiempo con estos; 6) orgullo nacional, el espectador asocia los triunfos de un competidor con el éxito del país del que proviene el deportista; 7) adoración, los espectadores se sienten atraídos hacia deportistas que parecen imbatibles; 8) violencia, la rudeza asociada a los deportes de combate podría ser un atractivo particular para los espectadores; 9) interés por el deporte, los asistentes acuden a ver las competiciones porque les gusta el judo como deporte; 10) interés por el competidor, los judocas a menudo luchan representando a diferentes organizaciones, pero los aficionados pueden haber acudido al evento para ver a sus competidores favoritos; y 11) interés por la organización, este motivo contempla la posibilidad de que los espectadores podrían seguir los eventos de la organización que los promueve. En el estudio de Kim et al. (2009) se analizaban doce motivos, pero debido a que en las competiciones de judo en España la entrada es gratuita y no se realizan apuestas, se ha descartado el factor económico como un motivo para la asistencia.

- Experiencias previas y preferencias (4 ítems): dos preguntas cerradas y dos preguntas abiertas, todas previamente codificadas y basadas en las aportaciones de Kim et al. $(2009,2008)$. En cuanto a experiencias previas en el judo, se busca conocer cómo el espectador conoció este deporte, y si había asistido o no a competiciones de judo con anterioridad. Por lo que respecta a sus preferencias, se pregunta sobre el evento de judo favorito del espectador y su categoría de peso preferida.

- Comportamientos en torno a los medios de comunicación (2 ítems): dos preguntas cerradas adaptadas a partir de Kim et al. $(2009,2008)$ para conocer si los asistentes a eventos de judo siguen este deporte o no a través de diferentes medios de comunicación, tales como la prensa, la televisión o internet.

Asistencia a eventos deportivos (2 ítems): mediante dos preguntas cerradas basadas en el trabajo de García y Llopis (2010), se pretende averiguar la asiduidad (frecuentemente, de vez en cuando, pocas veces o nunca) con la que los encuestados acuden a espectáculos deportivos en general, así como si han pagado o no por hacerlo en el último año.

- Hábitos de práctica deportiva (6 ítems): esta información, obtenida a partir de preguntas cerradas que se han adaptado del estudio de García y Llopis (2010), descubre en qué medida los espectadores de eventos de judo están vinculados con la práctica de este mismo deporte, así como con qué frecuencia, carácter y nivel de asociacionismo lo hacen.

En lo que respecta a la calidad de los instrumentos en los que se ha basado el cuestionario utilizado para el presente estudio, el trabajo de García y Llopis (2010) no hace referencia a aspectos como su fiabilidad y validez, si bien debe tenerse en cuenta que ha sido dirigido por profesionales del ámbito sociológico con una dilatada experiencia en el campo de la actividad física y el deporte. Asimismo, los autores desarrollaron dicho trabajo vinculados al Centro de Investigaciones Sociológicas, entidad dependiente del Ministerio de Presidencia de España con un amplio recorrido en la elaboración de encuestas de ámbito estatal con grandes muestras. Con todo lo expuesto anteriormente, cabe suponer que el instrumento utilizado por García y Llopis (2010) tuvo la suficiente calidad como para ser adaptado en el presente estudio. Por otro lado, los ítems provenientes del trabajo de Kim et al. (2009) fueron estimados fiables dado que, en el análisis de la consistencia interna, los coeficientes del alfa de Cronbach superaron la referencia que autores como Nunnaly y Bernstein (1994) consideran aceptable $(0,70)$. 


\subsection{Procedimiento}

El cuestionario requiere ser respondido de forma individualizada, con bolígrafo y papel facilitados en el mismo emplazamiento en el que se celebra el evento estudiado. Los encuestadores se desplazaron a los lugares de celebración de las competiciones seleccionadas, y establecieron aleatoriamente las áreas de gradas en las que se pediría a los asistentes su participación en el estudio. Los encuestadores se presentaron a los asistentes y explicaron los objetivos perseguidos por el estudio, en qué consiste el cuestionario y el tiempo necesario para su cumplimentación. Del mismo modo, en base a criterios éticos de la investigación, se hizo incidencia en que los documentos eran anónimos, no comportaban riesgos asociados y podían ser devueltos sin en cualquier momento. Una vez los espectadores accedían, se les proporcionó un bolígrafo y un cuestionario por persona, y fueron rellenados de forma autónoma.

\subsection{Muestra}

Debido a que no es posible obtener una muestra representativa del total de espectadores de eventos de judo absoluto celebrados en España, se optó por el procedimiento de muestreo no aleatorio mediante la elección de casos típicos: los espectadores asistentes a tres competiciones de judo absoluto, de diferentes niveles competitivos (local-autonómico y estatal).

Debido a que desde la RFEJYDA se nos confirmó que nunca se ha llevado la cuenta de los asistentes que acuden a sus eventos, nos valimos del cálculo estadístico para determinar el tamaño de nuestra muestra. Para un nivel de confianza del 90\%, el tamaño final de la muestra para nuestro estudio fue de 270 personas que asisten a ver eventos de judo absoluto en España. Finalmente, se consiguió la participación de las 270 personas previstas en las tres competiciones estudiadas.

En cuanto al criterio de selección de asistentes, se escogieron sectores de gradas al azar y se pidió colaboración a todos los asistentes de dichos sectores. Si bien se dieron casos puntuales en los que se negó la participación en nuestro estudio, la mayor parte de los asistentes preguntados accedieron a cumplimentar el cuestionario.

\subsection{Análisis de datos}

Para este estudio se realizó un análisis cuantitativo de los datos. Las respuestas relativas a preguntas cerradas fueron codificadas. Por su parte, las respuestas de las preguntas abiertas también fueron codificadas para identificar características que pudiesen ser analizadas cuantitativamente. Para ciertas dimensiones, se analizaron estadísticos descriptivos en los que se contemplan frecuencias y porcentajes. Es el caso de las dimensiones socio-demografía, experiencias previas, preferencias, comportamiento con los medios de comunicación, asistencia a eventos deportivos y hábitos de práctica deportiva. En el caso de la dimensión motivos, se realizó una comparación de valoraciones medias entre sexos mediante una prueba $U$ de Mann Whitney, debido a que la distribución resultó ser no-normal. Por otro lado, se comparó el nivel de práctica deportiva de nuestra muestra con el de la población analizada en el estudio de García \& Llopis (2010). No se pudo contrastar a través una prueba chi cuadrado, dado que no se pudo disponer de la base de datos utilizada por los autores mencionados previamente. Es por este motivo que la comparación se realizó por una prueba $Z$ de proporciones. Todos los cálculos estadísticos fueron realizados mediante el programa IBM SPSS Statistics 22.

\section{Resultados}

\subsection{Perfil del asistente a competiciones de judo}

En este apartado se muestran los resultados obtenidos en relación a la primera pregunta de investigación: ¿Cuál es el perfil del asistente a competiciones de judo absoluto en España? Dichos resultados están organizados en las dimensiones socio-demografía, motivos, experiencias previas, preferencias y comportamiento con los medios de comunicación.

La Tabla 1 muestra los perfiles mayoritarios de asistentes, de acuerdo con las variables correspondientes a la dimensión socio-demografía, en términos absolutos y en función del género 
de los participantes. Puede observarse que 157 asistentes eran hombres (58,1\% sobre el total), mientras que las mujeres sumaron 113 personas (41,9\%). Los asistentes de entre 45-54 años de edad fueron los más numerosos $(n=70 ; 25,9 \%)$, seguidos por los grupos de edad de 35-44 años ( $n$ $=55 ; 20,4 \%)$, y $25-34$ años $(n=43 ; 15,9 \%)$. Por otra parte, en cuanto al estado civil de quienes participaron en el estudio, puede observarse que aproximadamente la mitad de los encuestados eran solteros ( $n=133 ; 49,3 \%)$, seguidos por los casados $(n=124 ; 45,9 \%)$. En lo respectivo al nivel de estudios, los asistentes con titulación universitaria representaron la mayor parte de las personas encuestadas ( $n=105 ; 38,9 \%)$, seguidos de quienes habían completado estudios de formación profesional $(n=67 ; 24,8 \%)$ y de educación secundaria $(n=64 ; 23,7 \%)$. Pasando a la situación laboral de los participantes en el estudio, la mayor parte se encontraban en situación laboral activa ( $n=175 ; 64,8 \%)$, mientras que el segundo puesto en orden de representatividad estaba ocupado por los estudiantes ( $n=68 ; 25,2 \%)$. Por último, se observa que los encuestados de nacionalidad española representan casi la totalidad de los encuestados $(n=254 ; 94,1 \%)$.

Tabla 1. Características sociodemográficas de los asistentes a competiciones de judo absoluto. (Fuente: elaboración propia).

\begin{tabular}{|c|c|c|c|c|c|c|c|}
\hline \multirow{2}{*}{ Variable } & \multirow{2}{*}{ Categorías } & \multicolumn{4}{|c|}{ Género } & \multirow{2}{*}{ Total } & \multirow{2}{*}{$\%$} \\
\hline & & Hombre & $\%$ & Mujer & $\%$ & & \\
\hline \multirow{8}{*}{ Edad } & $<15$ años & 10 & $6,4 \%$ & 10 & $8,8 \%$ & 20 & $7,4 \%$ \\
\hline & 15-17 años & 4 & $2,5 \%$ & 3 & $2,7 \%$ & 7 & $2,6 \%$ \\
\hline & 18-24 años & 27 & $17,2 \%$ & 14 & $12,4 \%$ & 41 & $15,2 \%$ \\
\hline & 25-34 años & 22 & $14,0 \%$ & 21 & $18,6 \%$ & 43 & $15,9 \%$ \\
\hline & 35-44 años & 38 & $24,2 \%$ & 17 & $15,0 \%$ & 55 & $20,4 \%$ \\
\hline & 45-54 años & 37 & $23,6 \%$ & 33 & $29,2 \%$ & 70 & $25,9 \%$ \\
\hline & 55-64 años & 15 & $9,6 \%$ & 15 & $13,3 \%$ & 30 & $11,1 \%$ \\
\hline & $65 y+a n ̃ o s$ & 4 & $2,5 \%$ & 0 & $0,0 \%$ & 4 & $1,5 \%$ \\
\hline Total & & 157 & $100 \%$ & 113 & $100 \%$ & 270 & $100 \%$ \\
\hline \multirow{3}{*}{ Estado civil } & Soltero/a & 76 & $48,4 \%$ & 57 & $50,4 \%$ & 133 & $49,3 \%$ \\
\hline & Casado/a & 75 & $47,8 \%$ & 49 & $43,4 \%$ & 124 & $45,9 \%$ \\
\hline & Divorciado/a & 6 & $3,8 \%$ & 7 & $6,2 \%$ & 13 & $4,8 \%$ \\
\hline Total & & 157 & $100 \%$ & 113 & $100 \%$ & 270 & $100 \%$ \\
\hline \multirow{4}{*}{ Nivel de estudios } & Educación Primaria & 22 & $14,0 \%$ & 12 & $10,6 \%$ & 34 & $12,6 \%$ \\
\hline & Educación Secundaria & 36 & $22,9 \%$ & 28 & $24,8 \%$ & 64 & $23,7 \%$ \\
\hline & Formación Profesional & 45 & $28,7 \%$ & 22 & $19,5 \%$ & 67 & $24,8 \%$ \\
\hline & Universitarios & 54 & $34,4 \%$ & 51 & $45,1 \%$ & 105 & $38,9 \%$ \\
\hline Total & & 157 & $100 \%$ & 113 & $100 \%$ & 270 & $100 \%$ \\
\hline \multirow{4}{*}{ Situación laboral } & Estudiante & 36 & $22,9 \%$ & 32 & $28,3 \%$ & 68 & $25,2 \%$ \\
\hline & Trabajador/a & 102 & $65,0 \%$ & 73 & $64,6 \%$ & 175 & $64,8 \%$ \\
\hline & Parado/a & 14 & $8,9 \%$ & 7 & $6,2 \%$ & 21 & $7,8 \%$ \\
\hline & Jubilado/a & 5 & $3,2 \%$ & 1 & $0,9 \%$ & 6 & $2,2 \%$ \\
\hline Total & & 157 & $100 \%$ & 113 & $100 \%$ & 270 & $100 \%$ \\
\hline \multirow{2}{*}{ Nacionalidad } & Española & 145 & $92,4 \%$ & 109 & $96,5 \%$ & 254 & $94,1 \%$ \\
\hline & Otra & 12 & $7,6 \%$ & 4 & $3,5 \%$ & 16 & $5,9 \%$ \\
\hline Total & & 157 & $100 \%$ & 113 & $100 \%$ & 270 & $100 \%$ \\
\hline
\end{tabular}

Por otra parte, el Gráfico 1 permite apreciar que el motivo con valoraciones más altas a la hora de acudir a eventos de judo fue la calidad estética (5,93 puntos de media en hombres, 5,89 en mujeres), mientras que el que obtuvo una valoración más baja es la violencia $(1,54$ puntos en hombres, 1,48 en mujeres). Estos resultados coincidieron para ambos sexos, a excepción de la variable drama, en la que los hombres obtuvieron puntuaciones superiores a los de las mujeres $(U=$ 7561,00; $p=0,038)$.

En cuanto a la dimensión de experiencias previas, 143 de las personas encuestadas (53\% del total) descubrieron el judo a través de miembros de su familia, mientras que 54 de ellas (20\%) afirmaron haber conocido este deporte a través de amigos y/o conocidos. Hasta 35 personas (13\%) descubrieron el judo en su escuela o centro de estudios, 12 encuestados $(4,4 \%)$ lo hicieron gracias a los medios de comunicación, y 10 asistentes $(3,7 \%)$ por otras vías. Las 16 personas restantes $(5,9 \%)$ no lo recuerdan o bien no respondieron a esta cuestión. Por otro lado, 232 participantes 
$(85,9 \%)$ de este estudio habían acudido con anterioridad a otras competiciones de judo frente a las 38 personas $(14,1 \%)$ que asistían a uno de estos eventos por primera vez.

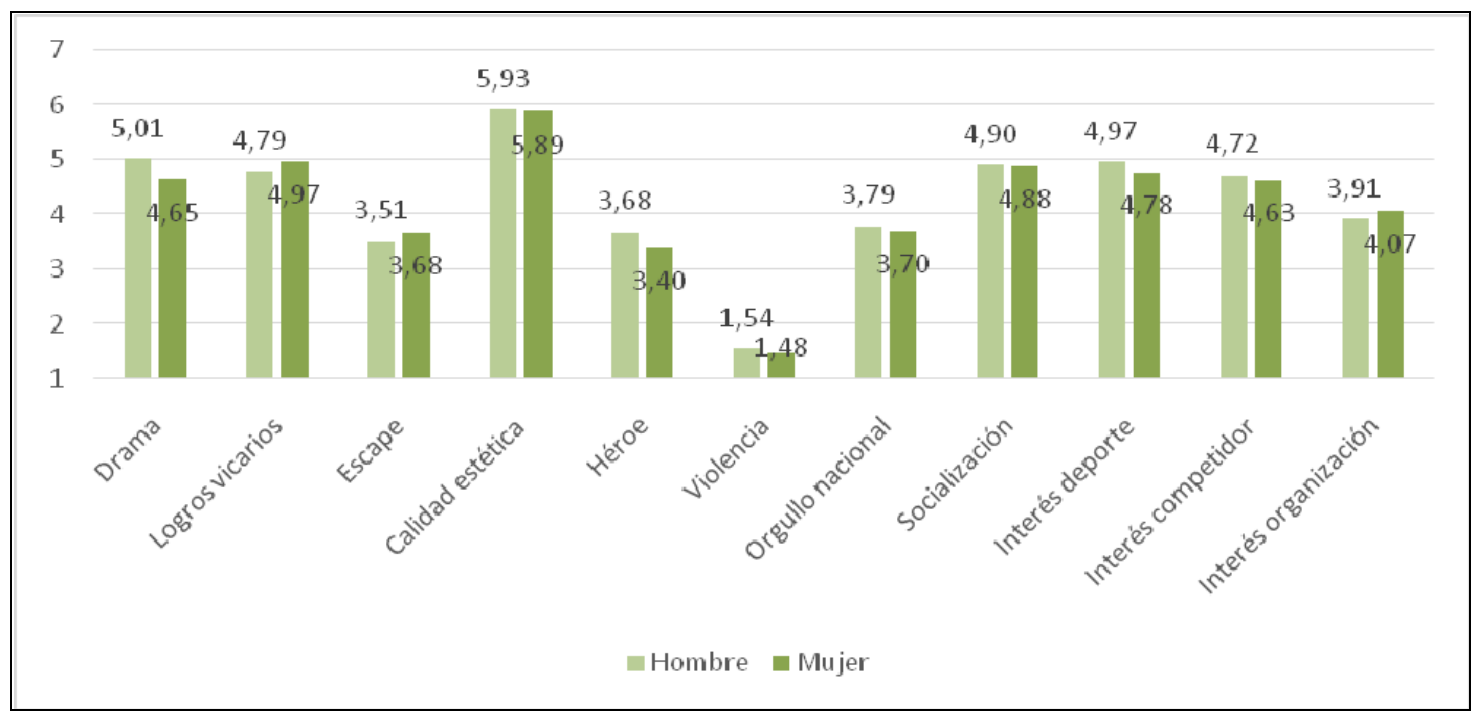

Gráfico 1. Valoración media de motivos por sexo. (Fuente: elaboración propia).

En lo respectivo a la dimensión que trata las preferencias, la mayor parte de las personas que colaboraron en esta investigación se inclinaron por las competiciones de nivel nacional $(35,6 \%)$, si bien también es significativo destacar la suma de personas que prefirieron competiciones internacionales en general $(20,4 \%)$ y aquellas que valoraron concretamente los Juegos Olímpicos $(13,3 \%)$. Hasta 12 participantes del estudio $(4,4 \%)$ señalaron su preferencia por otros tipos de eventos, mientras que $71(26,3 \%)$ no respondieron o bien no se inclinaron por ninguna de las opciones propuestas. Por otra parte, más de la mitad de los encuestados mostraron su preferencia por combates protagonizados por competidores de pesos semiligeros y medios (Tabla 2).

Tabla 2. Frecuencias y porcentajes para la variable categoría de peso favorita. (Fuente: elaboración propia).

\begin{tabular}{lcccc}
\hline Categoría de peso & Masculina & $\mathbf{\%}$ & Femenina & \% \\
\hline No sabe / No contesta & 64 & $23,7 \%$ & 72 & $26,7 \%$ \\
Superligeros (masculino hasta 60kg / femenino hasta 48kg) & 23 & $8,5 \%$ & 24 & $8,9 \%$ \\
Ligeros (masculino hasta 66kg / femenino hasta 52kg) & 28 & $10,4 \%$ & 41 & $15,2 \%$ \\
Semiligeros (masculino hasta 73kg / femenino hasta 57kg) & 57 & $21,1 \%$ & 46 & $17,0 \%$ \\
Medios (masculino hasta 81 kg / femenino hasta 63kg) & 52 & $19,3 \%$ & 52 & $19,3 \%$ \\
Semipesados (masculino hasta 90kg / femenino hasta 70kg) & 21 & $7,8 \%$ & 19 & $7,0 \%$ \\
Pesados (masculino hasta 100kg / femenino hasta 78kg) & 10 & $3,7 \%$ & 9 & $3,3 \%$ \\
Superpesados (masculino más 100kg/femenino más 78kg) & 15 & $5,6 \%$ & 7 & $2,6 \%$ \\
\hline Total & 270 & $100 \%$ & 270 & $100 \%$ \\
\hline
\end{tabular}

Por último, los datos referentes al comportamiento con los medios de comunicación muestran que la mayor parte de los encuestados $(n=174 ; 64,4 \%)$ seguían las noticias generadas por el judo en internet o prensa. Por otra parte, los participantes que veían eventos de judo en televisión o a través de internet ascendieron a 208, esto es, el $77 \%$ del total.

\subsection{Práctica de judo y contexto social de práctica de judo}

Este apartado expone los resultados vinculados a la segunda y tercera preguntas de investigación planteadas: ¿Qué proporción de practicantes de judo hay entre el público asistente a competiciones de judo absoluto en España? y ¿Cuán es el contexto social de práctica de los practicantes de judo que hay entre el público asistente a competiciones de judo absoluto en España? Estos resultados se muestran en función de las dimensiones asistencia a eventos deportivos y hábitos de práctica deportiva. Además, se muestran datos cruzados que facilitan la comprensión y proporcionan una respuesta más precisa a las preguntas planteadas. 
La información que se refiere a la dimensión de asistencia a eventos deportivos pone de manifiesto que 80 encuestados (29,6\% del total) acudieron frecuentemente a eventos deportivos a lo largo del último año, y 131 (48,5\%) lo hicieron de vez en cuando. En cambio, las personas que afirmaron haber asistido pocas veces sumaron 54 (20\%), mientras que $5(1,9 \%)$ expresaron no haber asistido a ningún evento deportivo. Por otro lado, cabe señalar que más de la mitad de los participantes en el estudio $(n=147 ; 54,4 \%)$ habían pagado por acudir a ver un evento deportivo a lo largo del último año.

En cuanto a los resultados para la dimensión de práctica deportiva, cerca de la mitad de los encuestados $(n=122 ; 45,2 \%)$ afirman practicar varios deportes. Junto con las 84 personas que practican un solo deporte $(31,1 \%)$, conforman más de tres cuartas partes de los participantes en este estudio, frente a los $64(23,7 \%)$ que no realizan ninguna actividad deportiva. En lo respectivo a la variable práctica de judo, se contemplaron como 'válidos' los encuestados que practicaban uno o varios deportes (206 personas en total), y como 'perdidos' a las personas que no practicaban ningún deporte (64 encuestados). Cerca de la mitad de los participantes válidos analizados ( $n=$ $101 ; 49 \%)$ expresaron que practicaban judo en la actualidad, mientras que 54 (26,2\%) lo habían hecho en el pasado. En cambio, 51 personas $(24,8 \%)$ nunca habían practicado judo.

A partir de aquí y hasta el fin de la exposición de resultados relativos a la dimensión de práctica deportiva, las variables que se muestran a continuación (frecuencia de práctica de judo, asociacionismo, carácter de la práctica de judo y licencia federativa) únicamente tuvieron en cuenta como 'válidos' los 101 participantes que afirmaron practicar judo en la actualidad, mientras que los 169 encuestados restantes no se contemplaron y contaron como 'perdidos'.

Los datos referentes a la variable frecuencia de práctica de judo muestran que 65 de los encuestados (64,4\%) realizaban tres o más entrenamientos semanales, y 23 (22,8\%) judocas practicaban entre una y dos veces por semana. Un total de 13 practicantes de judo $(12,9 \%)$ afirmaron entrenar con menor frecuencia. En cuanto al tipo de organización deportiva a la que pertenecían los encuestados que practicaban judo actualmente, 49 de ellas $(48,5 \%)$ lo hacían en un club privado. Hasta 26 personas $(25,7 \%)$ practicaban en un gimnasio o entidad similar, mientras que $24(23,8 \%)$ entrenaban en una asociación deportiva municipal. Apenas dos de los encuestados (2\%) afirman practicar este deporte en una asociación deportiva privada.

Por lo que respecta al carácter competitivo de la práctica de judo, 40 de los practicantes $(39,6 \%)$ hacían judo sin preocuparse por competir, y 12 de estos encuestados (11,9\%) competían con amigos por diversión. Por otra parte, hasta 49 personas competían en torneos, 21 de ellas (20,8\%) lo hacía a nivel local y/o autonómico, y 28 (27,7\%) a nivel nacional y/o internacional.

Por último, más de tres cuartas partes de los practicantes de judo $(n=79 ; 78,2 \%)$ poseían licencia federativa, mientras que 22 participantes $(21,8 \%$ del total), no tenían licencia expedida por la RFEJYDA.

Al cruzar las variables práctica deportiva y asistencia a eventos de judo, se observa que, si bien todos los grupos de personas tienden a haber asistido previamente a eventos de judo, esta tendencia es más destacada entre los que practican uno o varios deportes (ver Gráfico 2). Hasta 110 personas $(40,7 \%$ del total) que practicaban varios deportes afirmaron haber asistido anteriormente a competiciones de judo. Por otro lado, la proporción de personas que practicaban uno o más deportes en nuestra muestra fue superior $(76,3 \%)$ a la de la población en general $(43,0 \%)$, de acuerdo con datos de García y Llopis (2010). Esta diferencia es significativa $(z=11,05$; $p<0,01)$.

En cuanto al vínculo entre práctica de judo y asistencia a eventos de judo, tomamos como 'válidos' las 206 personas practicantes de uno varios deportes. Hasta 91 practicantes de judo en activo $(44,2 \%)$ habían asistido previamente a eventos de este deporte, frente a los 10 practicantes $(4,8 \%)$ que no habían acudido con anterioridad. En el caso quienes habían practicado judo en el pasado, 48 encuestados $(23,3 \%)$ respondieron que habían asistido a competiciones de judo antes, mientras que $6(2,9 \%)$ no lo habían hecho hasta la fecha. Por último, hasta 42 de los practicantes de deporte no vinculados con el judo $(20,4 \%)$ respondieron que habían acudido anteriormente a eventos de judo, contrastando con los $9(4,4 \%)$ que hasta entonces no habían visto un evento de esta disciplina deportiva. 


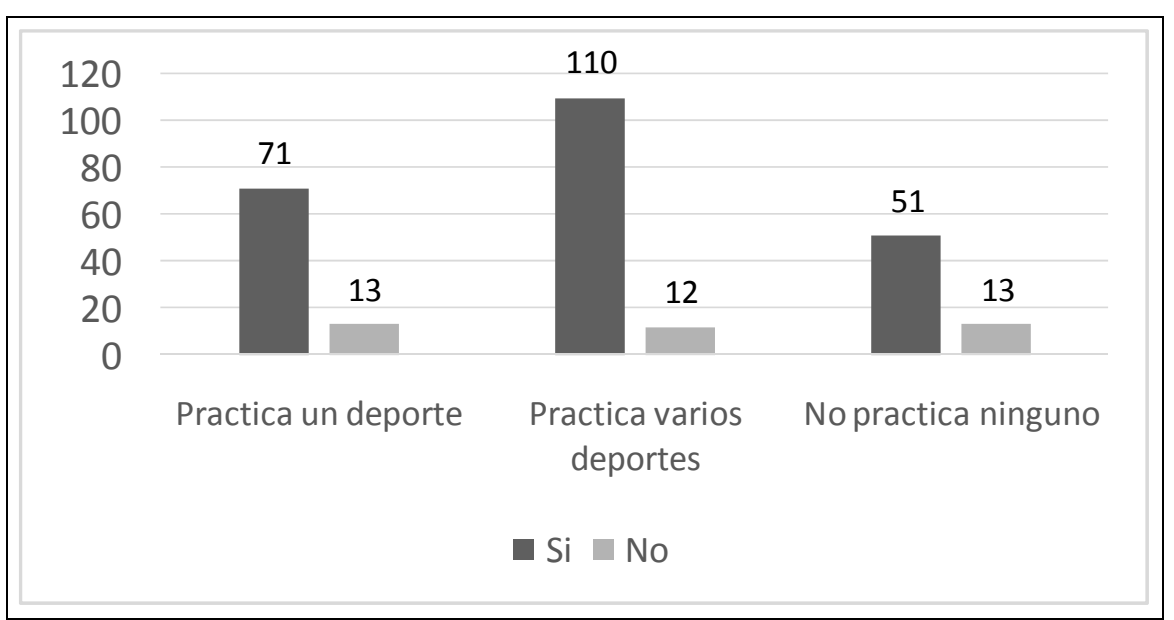

Gráfico 2. Relación entre las variables práctica deportiva y asistencia a eventos de judo. (Fuente: elaboración propia).

\section{Discusión}

El presente estudio expande el conocimiento existente en torno al análisis de los asistentes a eventos de artes marciales en general y del judo en particular, mostrando sus características y motivos que permitirían, por ejemplo, contribuir al desarrollo de estrategias de marketing en el sector. Por otro lado, contribuye a ampliar la información sobre las formas de consumir un deporte individual frente a los ampliamente estudiados deportes colectivos.

Este trabajo se centra en conocer el perfil de los asistentes a eventos de judo. Los espectadores en vivo de este deporte eran mayoritariamente hombres adultos, aspecto que contrasta con los hallazgos de Kim et al. $(2009,2008)$, en los que los asistentes a eventos de MMA eran también fundamentalmente hombres, pero más jóvenes. Esto puede ser debido a las estrategias de promoción utilizadas en un deporte emergente, que en ocasiones han venido de la mano de empresas promotoras tales como la Ultimate Fighting Championship (UFC). Al comparar esta información con los hallazgos de Molina (2002), el perfil de edad descrito en nuestro trabajo coincide con el de asistentes a deportes consolidados como el fútbol. Por otro lado, este estudio coincide con los trabajos de Kim et al., $(2009,2008)$ en cuanto a que la mayor parte de los asistentes analizados tenían estudios superiores de formación profesional o bien universitarios. Además, una gran proporción de las personas encuestadas se encontraban en situación laboral activa y habían pagado por asistir a eventos deportivos durante el último año. Estos resultados sugieren vías potenciales para aumentar los ingresos de la RFEJYDA, bien mediante la posibilidad de cobrar entrada en ciertos eventos, así como a través de anunciantes y/o patrocinadores que se puedan sentir atraídos por el perfil de los asistentes.

La calidad estética fue el motivo más valorado por los asistentes, seguida de la socialización y el interés por el deporte. El gran interés por los aspectos estéticos en este deporte individual concuerda con el trabajo de Wann et al. (2008), y puede deberse a la elevada proporción de practicantes y ex-practicantes de judo entre las personas encuestadas, así como el hecho de que la gran mayoría de personas que participaron en el estudio habían acudido con anterioridad a otras competiciones de este deporte. Por otro lado, es probable que entre los asistentes haya familiares y/o compañeros de club de los judocas que compiten en el evento, lo cual también ayuda a comprender por qué estos motivos fueron los mejor valorados. De igual modo, esta elevada vinculación y conocimiento del judo también podría explicar que la violencia fuese el motivo peor valorado, dado su código moral. Cabe señalar que la calidad estética y el interés por el deporte también se hallan entre los tres principales motivos mejor valorados por los asistentes a eventos de MMA analizados por Kim et al. (2008). A partir de esta coincidencia podría sugerirse que los asistentes a eventos de artes marciales y deportes de contacto son, por lo general, buenos conocedores de la disciplina que acuden a ver, y que aprecian especialmente la belleza del combate. Este trabajo coincide con autores como Hall et al. (2010) en aspectos como que la socialización es uno de los principales motivos para acudir a eventos de judo, en cambio no se ha valorado el efecto 
de otros aspectos que dichos autores consideran de importancia tales como las características de las instalaciones en las que se celebran los eventos deportivos.

La mayor parte de los encuestados expresaron que seguían eventos de judo a través de medios tales como la prensa, la televisión o internet, en consonancia con lo expuesto por autores como Mason (1999) o Smith (2008) en cuanto a los asistentes deportivos. Kim et al. $(2008,2009)$ coinciden en que el interés por el deporte y el seguimiento de eventos de dicha disciplina a través de los medios de comunicación cuando no es posible asistir en directo están estrechamente relacionados. Los resultados obtenidos parecen indicar una relación similar en el caso del judo en España.

En cuanto a la relación entre la asistencia y la práctica deportiva, se confirmó la hipótesis planteada junto con las preguntas de investigación, dado que la proporción de practicantes de deporte entre los asistentes a eventos de judo en España fue significativamente más elevada que la existente en la población general que asistía a eventos deportivos (García \& Llopis, 2010). En definitiva, los resultados de esta investigación se alinean con los trabajos que muestran una vinculación positiva entre práctica deportiva y asistencia a eventos deportivos (Sloan, 1989; Milne \& McDonald, 1999; Serrano, 1999; Thrane, 2001; Dawson \& Downward, 2009; Weed, 2009; García \& Llopis, 2010; Perényi, 2010; UK Sport, 2011; Lera \& Suárez, 2012). Esta vinculación positiva no solo existe a nivel de práctica deportiva general, sino también en cuanto a la práctica del deporte concreto que se ha estudiado en este estudio.

\section{Conclusiones}

Este estudio ha pretendido mostrar una primera aproximación sobre las características de los asistentes a eventos de judo en España, y sus resultados proporcionan sobre todo una imagen general acerca de cómo son los perfiles mayoritarios en nuestro objeto de estudio. Cabe destacar la elevada proporción de judocas en activo entre los asistentes a eventos de judo.

Los resultados obtenidos, junto con la información expuesta por investigaciones previas, sugieren que la mayor parte de los asistentes a eventos de judo son un público con un amplio conocimiento de este deporte. La información de este trabajo puede utilizarse por la RFEJYDA, así como por marcas vinculadas a las artes marciales y deportes de contacto, para desarrollar estrategias de marketing, campañas promocionales para la captación de asistentes, acciones de patrocinio, etcétera.

Cabe resaltar las limitaciones que condicionan la presente investigación y cuya resolución motivará el desarrollo de futuros trabajos. Consideramos que hubiera sido interesante añadir al instrumento de observación preguntas acerca de la vinculación de amistad y/o familiar de los encuestados con participantes de los diferentes torneos con el fin de comprobar la proporción de asistentes que viajan acompañando a los atletas y no únicamente como aficionados al deporte analizado. Bahia y Ávila (2011) afirman que el 30\% de los atletas analizados en su estudio viajó a un evento deportivo con amigos, el $40 \%$ con familiares, y el $30 \%$ con su comisión técnica. También se considera interesante poder llegar a una mayor muestra de encuestados con el objetivo de disponer de datos más fiables, dado que el índice de confianza para esta investigación ha sido del $90 \%$.Por último, creemos que ampliar este estudio a otras modalidades deportivas podría ser interesante para conocer los diferentes perfiles y hábitos de práctica de los asistentes a eventos de diferentes disciplinas, pudiéndose establecer también comparativas entre espectadores que acuden a ver diversos deportes.

Este estudio es pionero en cuanto a la aportación de conocimientos teóricos y datos empíricos acerca de los asistentes a competiciones de artes marciales y/o deportes de contacto en España, ofreciendo un marco metodológico para analizar sus perfiles. Esta investigación abre las puertas a seguir estudiando y ampliando nuestro conocimiento tanto en esta modalidad deportiva, como en otras disciplinas practicadas en el Estado español; algo que sin duda facilitará a los organismos reguladores información útil de cara a atraer más asistentes a sus eventos deportivos; así como que las empresas del sector puedan tomar decisiones estratégicas con una mayor seguridad.

Rev. Artes Marciales Asiát., 11(2), 99-111 2016 


\section{Agradecimientos}

Los autores quisiéramos reconocer la ayuda de las personas de la Real Federación Española de Judo y D.A. que han colaborado en este estudio, así como a las y los asistentes a competiciones de judo que han invertido parte de su tiempo en facilitarnos los datos sobre los que se sostiene la investigación. Asimismo, agradecemos a los encuestadores que, voluntariamente, participaron en la recogida de datos. Gracias también a la Dra. Ana Andrés, por su inestimable ayuda en el análisis de los datos. Finalmente, nuestra gratitud hacia la Dra. Núria Puig, por su apoyo a lo largo de todo el proceso.

\section{Referencias}

Benítez, J. \& Lacomba, B. (2012). Los avances en la valoración económica del deporte en Europa. Estudios de Economía Aplicada, 30(2), 1-18.

Burnett, J., Menon, A. \& Smart, D.T. (1993). Sports marketing: a new ball game with new rules. Journal of Advertising Research, 33(5), 21-35.

Consejo Superior de Deportes. (2015a). Licencias y Clubes 2015. Recuperado de http://www.csd.gob.es/csd/estaticos/asoc-fed/LicenciasyClubes2015.pdf

Consejo Superior de Deportes. (2015b). Datos económicos. Recuperado de http://www.csd.gob.es/csd/estaticos/asoc-fed/2015-6.Datos Economicos.pdf

Daniels, M. J., \& Norman, W. C. (2005). Motivations of equestrian tourists: an analysis of the colonial cup races. Journal of Sport \& Tourism, 10(3), 201-210. doi:10.1080/14775080500422494

Dawson, P., \& Downward, P. (2009). Participation, Spectatorship and Media Coverage in Sport. University of Bath.

Bahia, C.D.S., \& Ávila, M.A. (2011). Los eventos deportivos y el desarrollo turístico en Ilhéus-BahiaBrasil. Estudios y Perspectivas en Turismo, 20(1), 171-189.

Fink, J. S., Trail, G. T., \& Anderson, D. F. (2002). Environment factors associated with spectator attendance and sport consumption behaviour: Gender and team differences. Sport Marketing Quarterly, 11(1), 8-19.

Funk, D. (2008). Consumer Behaviour in Sport and Events: Marketing Action. Oxford: Elsevier.

Funk, D., Mahony, D., \& Ridinger, L. (2002). Characterising consumer motivation as individual difference factors: augmenting the Sport Interest Inventory (SII) to explain level of spectator support. Sport Marketing Quarterly, 11(1), 33-44.

García, M., \& Llopis, R. (2010). Encuesta sobre los hábitos deportivos en España. Madrid: Consejo Superior de Deportes y Centro de Investigaciones Sociológicas.

Greenwood, P. B., Kanters, M. A., \& Casper, J. M. (2006). Sport Fan Team Identification Formation in Mid-Level Professional Sport. European Sport Management Quarterly, 6(3), 253-265. doi:10.1080/16184740601095016

Hall, J., O'Mahony, B., \& Vieceli, J. (2010). An empirical model of attendance factors at major sporting events. International Journal of Hospitality Management, 29(2), 328-334. doi:10.1016/j.ijhm.2009.10.011

Havas Sports \& Entertainment (2013). La salud físico-deportiva de los españoles 2013. Recuperado de http://es.slideshare.net/jmance/hse-la-salud-fsicodeportiva-de-los-espaoles-2013

Kim, S., Andrew, D. P. S., \& Greenwell, T.G. (2009). An analysis of spectator motives and media consumption behaviour in an individual combat sport: Cross-national differences between American and South Korean Mixed Martial Arts fans. International Journal of Sports Marketing and Sponsorship, 10(2), 157-170.

Kim, S., Greenwell, T. C., Andrew, D. P. S., Lee, J., \& Mahony, D. F. (2008). An Analysis of Spectator Motives in an Individual Combat Sport: A Study of Mixed Martial Arts Fans. Sport Marketing Quarterly, 17(2), 109-119.

Lera, F., \& Rapún, M. (2006). Spectatorship and Participation in Sport: Two Different Leisure Models.

Recuperado de http://www.webmeets.com/files/papers/SAE/2006/115/FLerayMRapun.Oviedo2006.pdf

Lera, F., \& Suárez, M.J. (2012). Deporte activo y pasivo. ¿Una relación de conveniencia? Estudios de Economía Aplicada, 30(2), 489-512

Lines, G. (2007). The impact of media sport events on the active participation of young people and some implications for pedagogy. Sport, Education and Society, 12(4), 349-366. 
Mason, D.S. (1999). What is the sports product and who buys it? The marketing of professional sports leagues. European Journal of Marketing, 33(3/4), 402-418

Ministerio de Educación, Cultura y Deporte (2015). Encuesta de hábitos deportivos en España 2015. Recuperado de http://bit.ly/29qtBAb

Milne, G. R., \& McDonald, M. A. (1999). Sport Marketing: Managint the Exchange Process. Toronto: Jones \& Barlett.

Molina, F. (2002). Le profil sociologique des amateurs de football. Adhésions identitaires et fidélisation. Staps, (57), 69-84.

Perényi, S. (2010). On the fields, in the stands, in front of the TV - value orientation of youth based on participation in, and consumption of, sports. European Journal for Sport and Society, 7(1), 41-51.

Quispe, J. R. (2013). El valor de marca y el involucramiento deportivo en el futbol para generar estrategias en los clubes de futbol profesional. Recuperado de http://bit.ly/2oRWqsu

Quivy, R., \& Campenhoudt, L. (2005). Manual de investigación en Ciencias Sociales. México D.F.: Limusa.

Serrano, J.A. (1999). Prácticas y audiencias deportivas. Una exploración de la consistencia y dirección de sus relaciones. Revista Española de Investigaciones Sociológicas, (87), 101-134.

Shamir, B. \& Ruskin, H. (1984). Sports participation vs. Sport spectatorship. Two models of leisure behavior. Journal of Leisure Research, 16(1), 9-21.

Sloan, L. R. (1989). The motives of sport fans. En J.H. Goldstein (ed.), Sports, games and play. Social and psychology viewpoints (pp. 175-240). Hillsdale: Erlbaum Associates.

Smith, A. (2008). Introduction to sport marketing. Oxford: Elsevier.

Stone, G. P. (1971). American sports. Play and display. En E. Dunning (ed.), The sociology of sport (pp. 47-65). Londres: Frank Cass.

Thrane, C. (2001). Sport spectatorship in Scandinavia. A class phenomenon? International Review for the Sociology of Sport, 36(2), 149-163

Trail, G. T., \& James, J. (2001). Analysis of sport fan motivation scale. Journal of Sport Behaviour, 24(1), 108-128.

UK Sport. (2011). The inspirational effect of major sporting events. Recuperado de http://www.eventimpacts.com/pdfs/the inspirational impact of major sporting events.pdf

Vertonghen, J., \& Dortants, M. (2013). Report on the workshop "Organising, Managing and Regulating Martial Arts" during the 21 $1^{\text {st }}$ EASM conference. Revista de Artes Marciales Asiáticas, $8(2), 480-483$.

Wann, D. L. (1995). Preliminary validation of the Sport Fan Motivation Scale. Journal of Sport and Social Issues, 19(4), 377-396.

Wann, D. L., Grieve, F. G., Zapalac, R. K., \& Pease, D. G. (2008). Motivational Profiles of Sport Fans of Different Sports. Sport Marketing Quarterly, 17(1), 6-19.

Wasserberg, M. (2009). Understanding Sports Spectators' Motives for Attending Live Events: A Study of Darts Fans in the UK. Birkbeck: Sport Business Centre.

Weed, M. (2009). The potential of the Demonstration Effect to grow and sustain participation in sport. Canterbury Christ Church University: Centre for Sport, Physical Education \& Activity Research (SPEAR).

\section{Author's biographical data}

Pau Mateu Samblás (España), Graduado en Ciencias de la Actividad Física y del Deporte, Máster en Gestión Deportiva y cinto negro de judo 1을 DAN, actualmente es estudiante pre-doctoral en el INEFC de Barcelona y miembro del Grupo de Investigación Social y Educativa de la Actividad Física y el Deporte (GISEAFE). Entre sus áreas de interés se encuentran las organizaciones deportivas y su gestión, así como el mercado de trabajo en el deporte. E-mail: pmateu@gencat.cat

Eduard Inglés Yuba (España), Doctor en Ciencias de la Actividad Física del Deporte, es profesor de Actividades en el Medio Natural en el INEFC de Barcelona y docente en el Máster de Gestión Deportiva en el INEFC de Lleida. Su trabajo abarca áreas como las organizaciones deportivas y su gestión; el mercado de trabajo en el ámbito deportivo; y la relación entre deporte, territorio y desarrollo sostenible. E-mail: eduard.ingles@gencat.cat 